\title{
LUT
}

University

\section{Human-Centered Design Components in Spiral Model to Improve Mobility of Older Adults}

\author{
Khakurel Jayden, Porras Jari, Melkas Helinä
}

This is a Author's accepted manuscript (AAM) version of a publication

published by Springer, Cham

in Mobile Solutions and Their Usefulness in Everyday Life

DOI: $10.1007 / 978-3-319-93491-4 \_5$

Copyright of the original publication: (c) 2018 Springer Nature Switzerland AG

Please cite the publication as follows:

Khakurel J., Porras J., Melkas H. (2019) Human-Centered Design Components in Spiral Model to Improve Mobility of Older Adults. In: Paiva S. (eds) Mobile Solutions and Their Usefulness in Everyday Life. EAI/Springer Innovations in Communication and Computing. Springer, Cham

This is a parallel published version of an original publication. This version can differ from the original published article. 


\section{Metadata of the chapter that will be visualized online}

\begin{tabular}{|c|c|c|}
\hline Chapter Title & \multicolumn{2}{|c|}{$\begin{array}{l}\text { Human-Centered Design Components in Spiral Model to Improve } \\
\text { Mobility of Older Adults }\end{array}$} \\
\hline Copyright Year & \multicolumn{2}{|l|}{2019} \\
\hline Copyright Holder & \multicolumn{2}{|c|}{ Springer International Publishing AG, part of Springer Nature } \\
\hline \multirow[t]{7}{*}{ Corresponding Author } & Family Name & Khakure \\
\hline & Particle & \\
\hline & Given Name & Jayden \\
\hline & Suffix & \\
\hline & Organization & Lappeenranta University of Technology \\
\hline & Address & Lappeenranta, Finland \\
\hline & Email & Jayden.khakurel@lut.fi \\
\hline \multirow[t]{7}{*}{ Author } & Family Name & Porras \\
\hline & Particle & \\
\hline & Given Name & Jari \\
\hline & Suffix & \\
\hline & Organization & Lappeenranta University of Technology \\
\hline & Address & Lappeenranta, Finland \\
\hline & Email & jari.porras@lut.fi \\
\hline \multirow[t]{7}{*}{ Author } & Family Name & Melkas \\
\hline & Particle & \\
\hline & Given Name & Helinä \\
\hline & Suffix & \\
\hline & Organization & Lappeenranta University of Technology \\
\hline & Address & Lappeenranta, Finland \\
\hline & Email & helina.melkas@lut.fi \\
\hline Abstract & \multicolumn{2}{|c|}{$\begin{array}{l}\text { As humans grow older, their cognitive needs change more frequently } \\
\text { due to distal and proximal life events. Designers and developers need } \\
\text { to come up with better designs that integrate older users' needs in a } \\
\text { short period of time with more interaction with the users. Therefore, the } \\
\text { positioning of human end users in the center of the design itself is not } \\
\text { the key to the success of design artifacts while designing applications } \\
\text { for older adults to use a smartphone as a promising tool for journey } \\
\text { planner while using public transportation. This study analyzed the use } \\
\text { of human-centered design (HCD) components, the spiral model, and the } \\
\text { design for failure (DfF) approach to improve the interactions between } \\
\text { older users and designers/developers in gathering usability needs in the } \\
\text { concept stage and during the development of the app with short iterative } \\
\text { cycles. To illustrate the importance of the applied approach, a case study } \\
\text { with particular focus on older adults is presented. }\end{array}$} \\
\hline
\end{tabular}




\author{
Jayden Khakure, Jari Porras, and Helinä Melkas
}

\begin{abstract}
As humans grow older, their cognitive needs change more frequently due 6 to distal and proximal life events. Designers and developers need to come up with 7 better designs that integrate older users' needs in a short period of time with more 8 interaction with the users. Therefore, the positioning of human end users in the 9 center of the design itself is not the key to the success of design artifacts while 10 designing applications for older adults to use a smartphone as a promising tool 11 for journey planner while using public transportation. This study analyzed the use 12 of human-centered design (HCD) components, the spiral model, and the design 13 for failure (DfF) approach to improve the interactions between older users and 14 designers/developers in gathering usability needs in the concept stage and during the 15 development of the app with short iterative cycles. To illustrate the importance of 16 the applied approach, a case study with particular focus on older adults is presented. 17
\end{abstract}

\title{
5.1 Introduction
}

The proportion of the older age group is growing [1,2], and it is expected that in 19 upcoming years, many older adults in North America and Europe will travel using 20 their own transportation (i.e., with their own private cars) [3] because of the desire 21 to be autonomous. Ego enhancement, self-esteem, novelty, knowledge seeking, 22 relaxation, socialization, and cultural and historical factors were cited as motivation 23 for travel among older adults [4-6]. However, Rosenbloom and Ståhl (2003) point 24 out, "The growing automobility of an aging population poses environmental, safety, 25 mobility, and community design challenges to developed societies" (pp. 210). One 26 of the possible ways to reduce the automobility of an aging population is to 27 encourage the use of alternative modes of mobility, such as public transportation; 28 [7-9] point out, "public transport is important to older people's quality of life, 29

\footnotetext{
J. Khakure $(\square) \cdot$ J. Porras $\cdot$ H. Melkas

Lappeenranta University of Technology, Lappeenranta, Finland

e-mail: Jayden.khakurel@lut.fi; jari.porras@lut.fi; helina.melkas@lut.fi

(C) Springer International Publishing AG, part of Springer Nature 2019

S. Paiva (ed.), Mobile Solutions and Their Usefulness in Everyday Life,

EAI/Springer Innovations in Communication and Computing,

https://doi.org/10.1007/978-3-319-93491-4_5
} 
their sense of freedom and independence" (pp. 4334). However, journeys via public 30 transportation require travelers to (i) get engaged in a series of high-level activities 31 such as planning, waiting, and moving; (ii) comprehend, manipulate, and process 32 "essential navigation artifacts"; and (iii) accommodate unexpected situations due to 33 system failure or a user's own error [7]. Such requirements can be a major hurdle 34 for older adults in terms of accessibility, perceptions about safety, and management 35 of the associated informational complexity [10, 11] along with the consequent 36 high cognitive load. Responding to these challenges is possible by meeting the 37 older user's cognitive and physical access needs through smart technological tools, 38 thereby enabling them to use public transportation.

Among the many existing technologies, smartphones and installed applications 40 can be considered as promising tools that can support the effective use of graphical 41 user interfaces (GUIs), allowing users with visual impairments to customize their 42 phones to suit their particular needs [12]; manage mobility by providing transit 43 information and aiding navigation [13,14]; and improve the quality of life for older 44 adults $[15,16]$. However, in designing for older adults within public transportation 45 information technology (IT) environments, such as real-time transit and navigational 46 information, the requirements are not necessarily the same as those for other 47 categories of the population [11]. A major problem is that stakeholders have little 48 understanding of how to provide better touchscreen interface tools for older users, 49 since the de facto standards of basic operations on touchscreen-based smartphones, 50 which consist of tapping, dragging, and pinching, have only been adopted in the 51 last few years [17]; how to integrate audible, haptic, and visual feedback that is 52 responsive to older users' physical, cognitive, and mental capacities; and how to 53 design applications for failure caused by the device, the environment, and the user's 54 own action and supporting technology in a special context; [9, 18] state, "As the 55 system developers target bigger markets from their economic point of view, many 56 products do not address the requirements of specific groups of older people (e.g. 57 those with mobility difficulties)" (pp. 4334).

This research attempted to bridge the knowledge gap by undertaking human- 59 centered design (HCD) with the spiral model and the design for failure (DfF) 60 methodological approach within the public transportation information technology 61 (IT) environment using a smartphone application to support older users in managing 62 and improving their mobility. This approach, which was validated by the case study, 63 can help transit stakeholders to understand how smart mobile applications can meet 64 the mobility needs of older adults by identifying and analyzing single probable 65 points of failure based on interacting with older end users during all phases of the 66 design and development process. Accordingly, the objectives of this research were: 67

- To extend the HCD design approach by using a spiral model and DfF and 68 propose a design process for the development of applications targeted at older 69 adults in an effort to improve HCD's usefulness to application designers and 70 developers. Even though the results are solely based on a web and Android 71 platform, we believe that this design and development approach can be utilized in 72 


\section{Author's Proof}

5 Human-Centered Design Components in Spiral Model to Improve Mobility...

other application development processes while working with and for older adults 73 to enhance their quality of life.

- To illustrate the usefulness of HCD with a spiral model and DfF approach during 75 the design process with particular focus on older adults, a web platform and 76 smartphone-based application was developed. It lets older adult travelers prepare 77 their personalized journey via public transportation at their convenience and then 78 receive guidance throughout their journey.

Hence, to achieve the objective, this study aimed to answer the following research 80 questions:

RQ1: How does an approach that uses the human-centered iterative design and 82 development activities of application development with a spiral model and design 83 for failure improve the usability needs of older adult users?

RQ2: What types of usability issues related to device characteristics (i.e., hardware 85 and software) were discovered during the case study?

In the following section, we present related work, followed by the case study and 87 our methodology to validate our approach. Following this, we present an interaction 88 analysis framework in line with the case study, followed by the key findings, 89 limitations, and conclusion.

\subsection{Background Study}

In this section, we describe previous work to understand the impact of aging on 92 access to public transportation; technology; as well as the benefits of HCD, the 93 spiral model, and design for failure that researchers have discussed in recent years. 94

Regarding the impact of aging on technology and vice versa, [19] investigated the 95 factors that influence the use of user interfaces (UIs) among older users. Zajicek's 96 (2001) study showed that age can impact the interaction between older users and 97 the user interface. Further, Zajicek (2001) found that modalities such as speech 98 interaction are useful for older users with visual impairments. However, such 99 modalities had drawbacks for some older users in trying to follow lengthy output 100 messages. Zajicek (2001) concluded that older users' requirements may vary during 101 the design period due to tiredness, eyestrain, and decreasing abilities over time and 102 designers should take older users' changing abilities into consideration during the 103 design process. [20] attempted to understand how accuracy and performance vary 104 between younger and older users while using the same user interface. The study 105 involved experiments where performance was observed under three instructional 106 sets: accuracy (i.e., users were asked to focus on accuracy only); neutrality (i.e., 107 users were asked to focus on both speed and accuracy), and speed (i.e., users were 108 asked to focus on speed only). They concluded that aging has a significant negative 109 effect on performance and accuracy. [21] performed usability evaluation studies on 110 the user interface of a mobile application targeted at older users. The study produced 111 
a set of recommendations regarding navigation, interaction, and the visual design 112 aspects of smartphone application user interfaces.

With respect to the research on the requirements of the needs of older adults 114 in using public transportation, [22] pointed out that due to the impacts of aging, 115 older travelers have a variety of safety, personal security, flexibility, reliability, 116 and comfort concerns about public transit, even if it is physically accessible. [9] 117 conducted a study on the requirements of five different profiles/groups of older 118 people using the public transportation. The study found that easily accessible 119 information provision on public transport was important for all five different 120 profiles/groups of older people but that the required content and delivery of the 121 information varied between groups. [23] conducted a study with a sample of older 122 citizens $(75+$ years $)$ to understand their perceived travel opportunities. The study 123 concluded that more needs to be done with regard to accessibility and usability of 124 public transit for older people.

\subsection{Methodological Approach}

To be able to easily access public transportation, older adults will need to know how 127 to manage their tools, locate transit information, and navigate more effectively with 128 minimal cognitive needs. Therefore, when it comes to this target group of users, 129 we believe that it is not enough for stakeholders to decide what older adults will 130 consider an intuitive design. To gather the specific requirements for a user interface 131 and gain its adoption by older users, we considered an approach that uses human- 132 centered design (HCD) with a spiral model presented by Boehm [24] and design for 133 failure to be the foundation of this methodology.

Previous studies have incorporated human-centered design (HCD) to make 135 systems usable and useful by focusing on the users, their needs and requirements, 136 and by applying human factors/ergonomics and usability knowledge and techniques 137 [25]. Kujala (2003) [26] pointed out that user involvement has positive effects if the 138 user's role is carefully selected, especially during requirements elicitation and user 139 satisfaction. Kujala (2003) stated that "users may not be able to communicate their 140 precise requirements but they are able to explain their goals and how they approach 141 their tasks" (pp. 13). Similarly, Uzor et al. (2012) [27] demonstrated the impact of 142 older adults during the design process.

The results of their study showed that by participating in the design process, 144 older users can reveal some of the obstacles that designers may not be familiar 145 with, enabling them to resolve such obstacles in an early stage of the development 146 process. Ferreira et al. (2013) [28] conducted research on lowering the high levels of 147 nonadherence to medication in the older population by using a mobile application 148 that puts the user at the center of the design process. The study concluded that the 149 participation of older users from the initial phase and onward helped to generate 150 ideas, enabling the application to be shaped according to the users' needs and 151 capabilities through a continuous process of redesign. However, in the case of older 152 


\section{Author's Proof}

5 Human-Centered Design Components in Spiral Model to Improve Mobility...

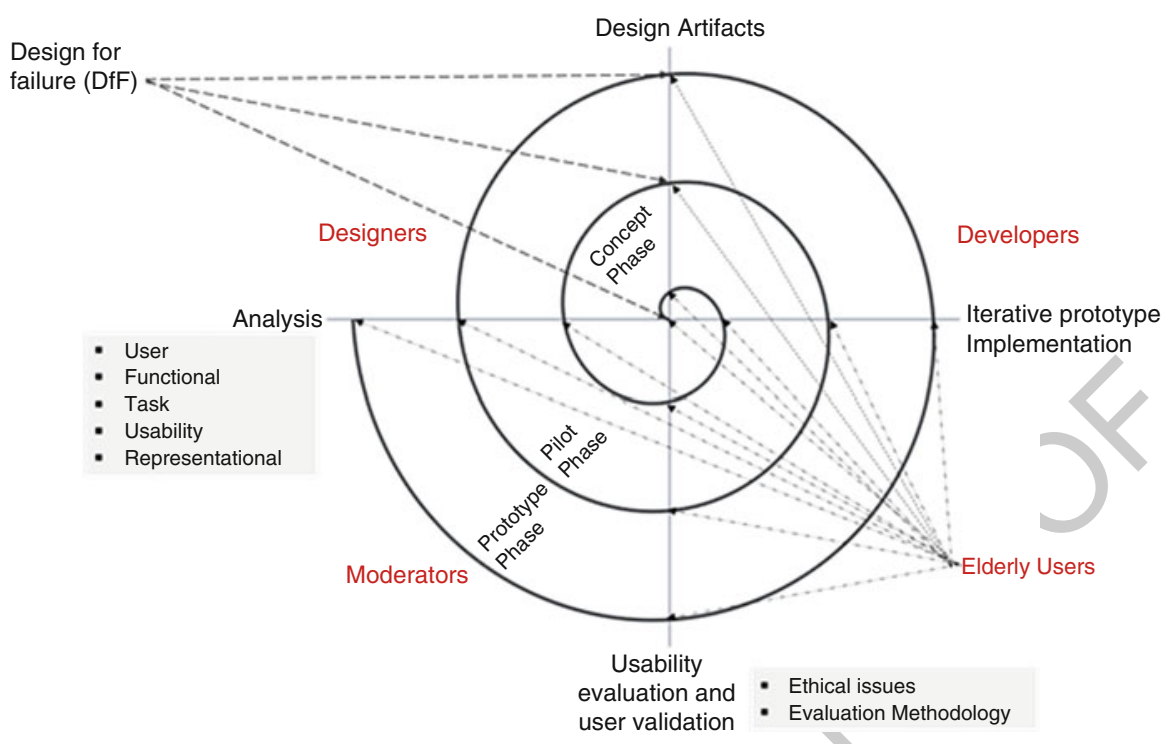

Fig. 5.1 HCD component in a spiral model with a design for failure framework

adults, the human-centered design approach has some challenges because older 153 users "may not be able to critique a design until it is far along in the development 154 process, which may be too late to make certain types of changes given time and 155 cost constraints" ([29], pp. 8). Such challenges may cause tremendous risks during 156 a project. Thus, to reduce the risk, we adopted a short iterative spiral model (i.e., 157 three distinct phases: the concept phase, the prototype phase, and the pilot phase) 158 and embedded HCD components (i.e., analysis, design artifacts, iterative prototype 159 implementation, and usability evaluation and user validation) (see Fig. 5.1). 160

Further, technology failures are inevitable during an application's design, devel- 161 opment, and implementation because of the complexity of the system. Similarly, 162 human errors while using devices or applications are inevitable because of the need 163 for a high cognitive load to learn new things. Such errors can have adverse effects on 164 older adults' use of a system during public transit. To reduce such adverse effects, 165 we implemented the DfF approach during the design process, which is common 166 in software design. This study applied the DfF framework presented by Carmien 167 (2017), meaning the system should be able to accommodate, which consists of 168 four basic types of failure events: (i) device failure, (ii) environmentally caused 169 failure, (iii) failure due to user action, and (iv) failure due to supporting technology 170 in special contexts.

To help illustrate the concepts discussed and present the results of our study, 172 we now present the proposed "Assistant" web platform and mobile application. [9] 173 point out, "open access data provision in the transport sector has a huge potential 174 to encourage dissemination of targeted traveler information using mobile apps 175 with the involvement of the private sector" (pp. 4342). Therefore, the aim of this 176 


\section{Author's Proof}

J. Khakure et al.

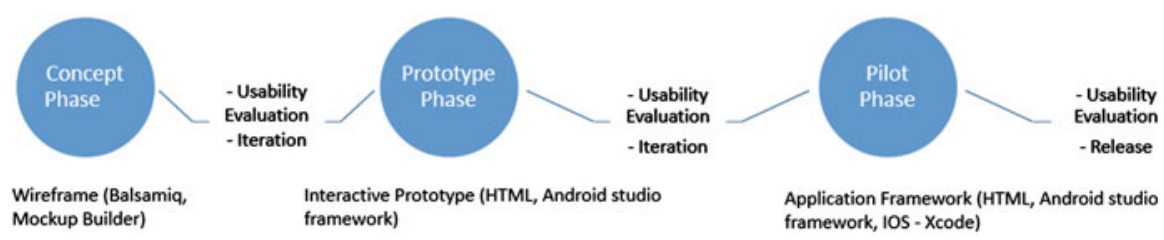

Fig. 5.2 Design iteration process

project was to develop a novel personalized and customized public transportation 177 trip planner for older adults with or without disabilities based on the open data. 178 The Assistant provides guidance on transfers when making multi-step journeys and 179 assistance in getting from the vehicle to the final destination. This allows Assistant 180 applications to accommodate expected human errors, increase accessibility, and 181 enable effective error handling. The structure of the Assistant system comprises 182 five main components:

- Web-based route editor platform: The backend maintains a database of public 184 transportation information (i.e., open data) from transit providers and other 185 customized and personalized user information for both primary and secondary 186 users. The Assistant's web interface allows users to create a route on a map, 187 view route calculations, and manage their profiles. Simultaneously, it monitors 188 the users' interactions with the system, checks data for errors, and provides 189 corrective support. Once personalized routes are created, push notification 190 messages are sent to the users' smartphone devices (see Fig. 5.2). 191

- Mobile Application: The Assistant's mobile application communicates with the 192 server asynchronously over the Internet and retrieves updated maps, schedules, 193 and telematics data.

\subsection{Analysis of Methodological Approach}

The following section describes how each HCD component was incorporated with 196 respect to the design of the Assistant application for older users within all three 197 distinct phases (concept, pilot, and prototype).

\subsubsection{Analysis}

A key component of HCD is analysis, which comprises multiple levels: users, 200 functions, tasks, and representations [30]. Rinkus et al. (2005) further explained, 201 "The user analysis level contributes to each of the levels of functional, task, 202 and representational analysis" (pp. 5). Since the system is designed for older 203 


\section{Author's Proof}

5 Human-Centered Design Components in Spiral Model to Improve Mobility...

users, we undertook usability as part of the components of analysis. Therefore, 204 in our approach, the user analysis level consisted of usability, functional, task, 205 and representational level. The following section provides a synopsis of how each 206 requirement in this level was extracted and compiled from the focus groups of older 207 users:

- User analysis: The elderly users' needs vary widely based on their physiological 209 and psychological characteristics, which influence the adoption and acceptation 210 of the system. This level focuses on identifying and analyzing the elderly users' 211 physiological and psychological characteristics, which help to design a system 212 that matches their needs, main difficulties, and limitations. Assistant, which is an 213 "on the go" system, consists of two separate users:

- Primary users: The older users' needs varied widely based on their physio- 215 logical and psychological characteristics, which influenced the adoption and 216 acceptance of the system. This level focused on identifying and analyzing the 217 older users' physiological and psychological characteristics, which helped to 218 design a system that matched their needs, main difficulties, and limitations. $\quad 219$

- Secondary users: Caregivers and/or relatives who may use different modules 220 of the "on the go" system to support primary users who may or may not have 221 reduced cognitive abilities.

- Usability analysis: A system that has older adults as its primary users requires 223 early usability analysis to bridge the gap between users and devices. Usability 224 can be defined as the ability of both primary and secondary users to interact 225 easily with the device and its characteristics (such as physical aspects and 226 software) when used under specified conditions to achieve specific goals [31]. 227 The requirements obtained from a usability analysis must be tangible and can 228 be verified and traced during development [32]. For this purpose, before starting 229 the functional analysis, we asked some older users to answer several questions 230 in order to collect their usability requirements. Based on the collected data and 231 the usability principles provided by Nicolle et al. (1999) [33], ISO/IEC (2001) 232 [36], Parhi et al. (2006) [34], and Creswell (2007) [35], the following system 233 requirements for the Assistant were obtained: it should be simple and user 234 friendly; the user interface (UI) should display only required functionalities; the 235 UI should be personalized based on user preferences due to cognitive abilities; 236 any error, warning, and other messages should be relevant and concise; the UI 237 should be designed with more legible fonts and larger font sizes; the UI should 238 follow all legibility guidelines relating to color contrast, lighting, font size, and so 239 on; the application should have visual and audible instructions and haptic signals; 240 and physical buttons should be utilized rather than touchscreens.

- Functional analysis is the process of identifying critical top-level domain 242 structures, goals, and inherent properties of the work domain that are largely 243 independent of implementation [30]. Rinkus et al. (2005) further stated that "it is 244 more abstract than task and representational analyses because it does not involve 245 
details of task processes and representation details" (pp. 6). The functional 246 analysis results established the following requirements:

- Route planning (safer and effective ways to plan and compose personalized 248 routes)

- Map accessibility annotation (provide a set of information about the essential 250 accessibility data, such as barriers, bus/metro stops, and public facilities 251 during route planning)

- Waypoint navigation (provide turn-by-turn guidance with waypoints for the 253 personalized routes on smartphone devices during the trip) 254

- Last kilometer navigation (navigate the user from the last transit stop to the 255 final destination using a map that consists of point-of-view maps and visual 256 waypoint support)

- User preferences (user's personal accessibility needs and preferences are 258 taken into account)

- Error detection and mediation (detects and accommodates human error as well 260 as the failure of system components, e.g., going off track, stolen phone, loss 261 of signal, success or failure of planned routes, etc.)

- Task analysis: Task analysis is the process of identifying the procedures and 263 actions to be carried out and the information to be processed to achieve the users' 264 task goals [30]. For the Assistant, we broke down the process into the following 265 steps based on each function, identified during the functional analysis phase: 266 high-level scenario, use case, test case, and tasks.

- Representational analysis: A representational analysis, which is based on the 268 representational effect [37], refers to a phenomenon that analyzes the isomorphic 269 restrictions that can have dramatic effects on the user's ability to accomplish 270 the tasks. The form of a representation can influence and sometimes determine 271 what information can be easily perceived, what processes are activated, and what 272 can be derived from the representation [30]. During this process, suitable com- 273 munication tools (e.g., smartphone devices, computer displays, and customized 274 original component manufacturer devices) with context display interfaces for the 275 given task for the specific set of users can improve the users' experiences overall. 276

Because of widespread smartphone device characteristics (i.e., physical 277 aspects and software) in the market, the limitations placed by the smartphone 278 device manufacturers on development platforms, and the users, tasks, usability, 279 and functional requirements, we chose to use an Android-based Samsung 280 smartphone. This smartphone allows users to install customized applications 281 and has a high-resolution display, customizable widget size, fewer limitations 282 in comparison to other OS development platforms, and device connectivity 283 functionalities such as Bluetooth. 


\section{Author's Proof}

5 Human-Centered Design Components in Spiral Model to Improve Mobility...

\subsubsection{Summary of User Requirements}

The following section summarizes the older users' requirements that need to be 286 taken into consideration on both the mobile and web.

Mobile: In general, the preference was text waypoints; audio of prompts was 288 desired; request to keep smartphone out of the way to diminish the possibility of 289 being stolen was preferred; design should take into account the role of dual glasses; 290 Bluetooth headset was the preferred output device by many; there was a need for 291 clarity on the waypoint page and audible prompts; fonts cannot be small; spoken 292 control of the system was preferred; widget size and appearance were preferred; 293 touchscreen response was preferred; switching between apps was required; and 294 users would like orientation information on the screen. 295

Web: Scale of the maps and street names must be readable; maps should have the 296 "you are here" indicator; word completion for addresses should be an option; a new 297 terminology should not be used; font size is important; text route instructions should 298 be included; and transfer of data from a web platform to a smartphone is required. $\quad 299$

\subsubsection{Design Artifacts}

Design is essentially a search process to discover an effective solution to a problem 301 [38], not only to provide a better user experience but also to establish the credibility 302 of proposed or existing systems. For our case study, we defined and designed 303 artifacts (i.e., architecture, components, modules, and user interfaces). As this 304 analysis focuses on improving the usability needs of older users, we skipped 305 the technical analysis and focused more on the design analysis to improve their 306 experiences.

During the analysis, it became clear that the user interface acted as a tool for 308 older users to interact with the system and required a particular set of features 309 to meet all the users' cognitive needs. Our first objective was to design a user- 310 friendly interface based on the specified requirements obtained from the analysis to 311 maximize usability and the user's experience. Therefore, early in the design process, 312 interviews and focus groups were held to derive a set of user requirements and 313 different tool kits were utilized (see Fig. 5.2).

Three initial designs were prepared and presented to a focus group of older users 315 to receive their feedback during the concept phase (see Fig. 5.3a). During the first 316 design, online tools "Balsamiq" and "Mockup Builder" " were used to create the 317 wireframe. The next iteration of the design cycle was triggered to improve and 318 introduce a new design based on users' feedback after usability evaluation where 319

\footnotetext{
${ }^{1}$ https://balsamiq.com/

${ }^{2}$ http://mockupbuilder.com/
} 


\section{Author's Proof}

J. Khakure et al.
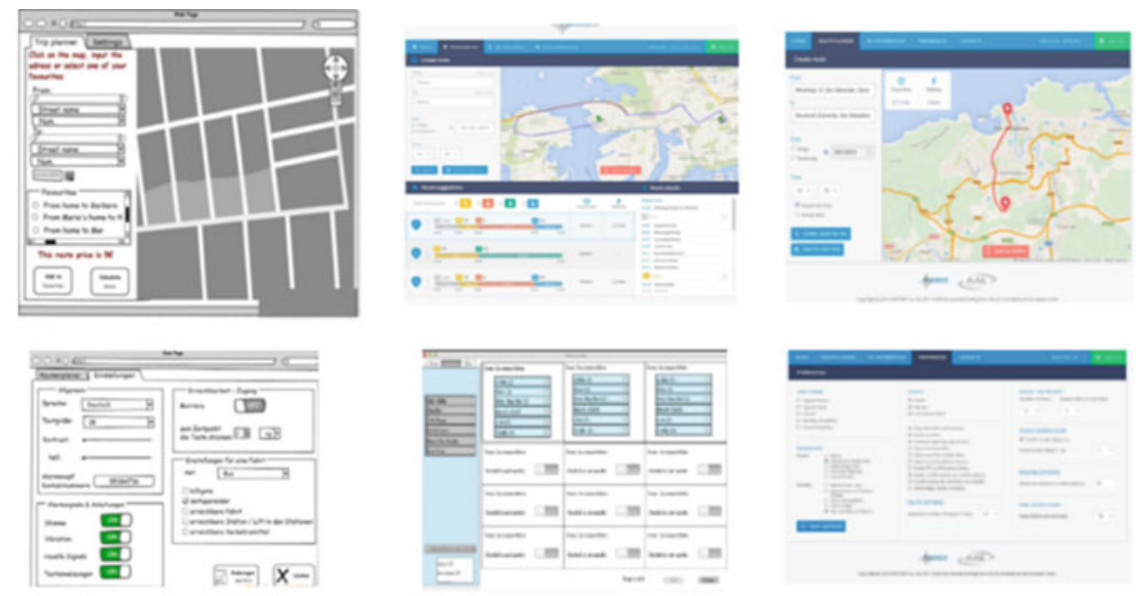

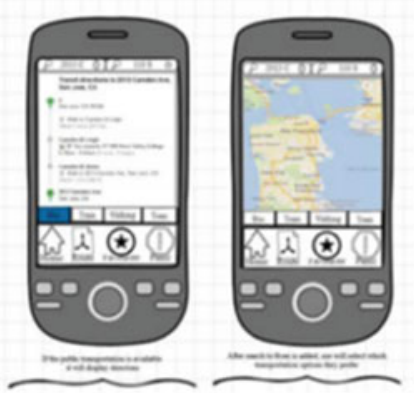

a

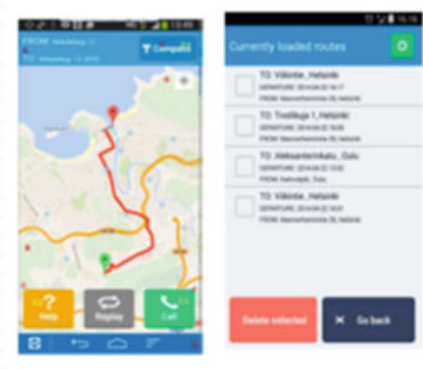

b
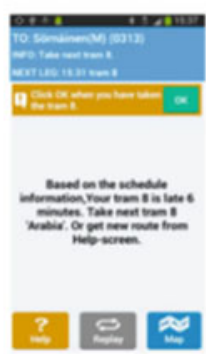

c

Fig. 5.3 Design iterations. (a) Concept phase. (b) Prototype phase. (c) Pilot phase

HTML was used to create the website and Android studio framework and their 320 libraries were utilized for smartphone application (see Fig. 5.3b). A final application 321 was presented and tested among the older adults (see Fig. 5.3c).

\subsubsection{Iterative Prototype Development and Implementation}

In the concept phase, the initial stage prototype was implemented using a wireframe 324 (see Fig. 5.4a, b) for both web and smartphone application based on the primary 325 user's requirements, which was useful in outlining the user interface.

During prototype phase, usability protocols were implemented to gather the 327 users' feedback on the mobile device itself. We identified a number of usability 328 issues such as the design and physical shape of the devices in the initial mock- 329 up, even though we followed the usability guidelines stated by Kobayashi et al. 330 


\section{Author's Proof}

5 Human-Centered Design Components in Spiral Model to Improve Mobility...

a
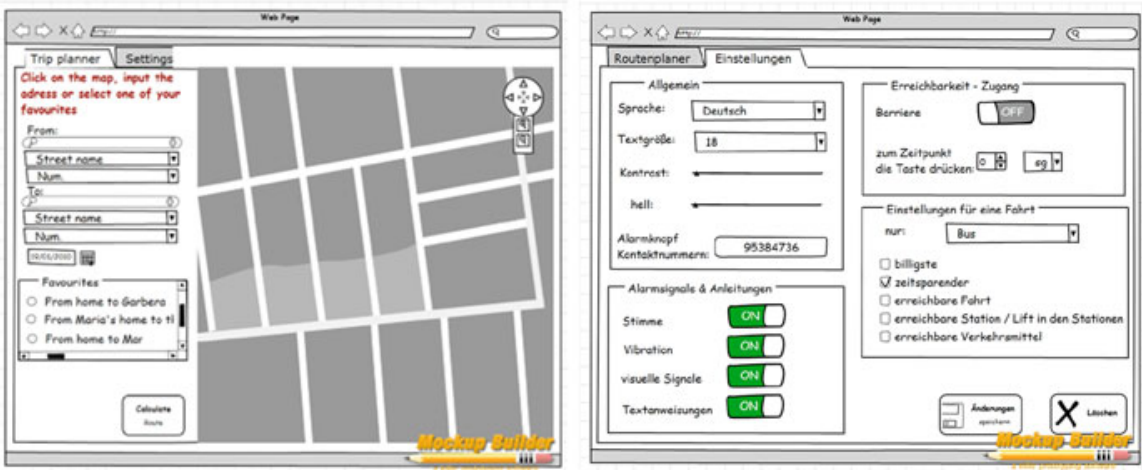

b
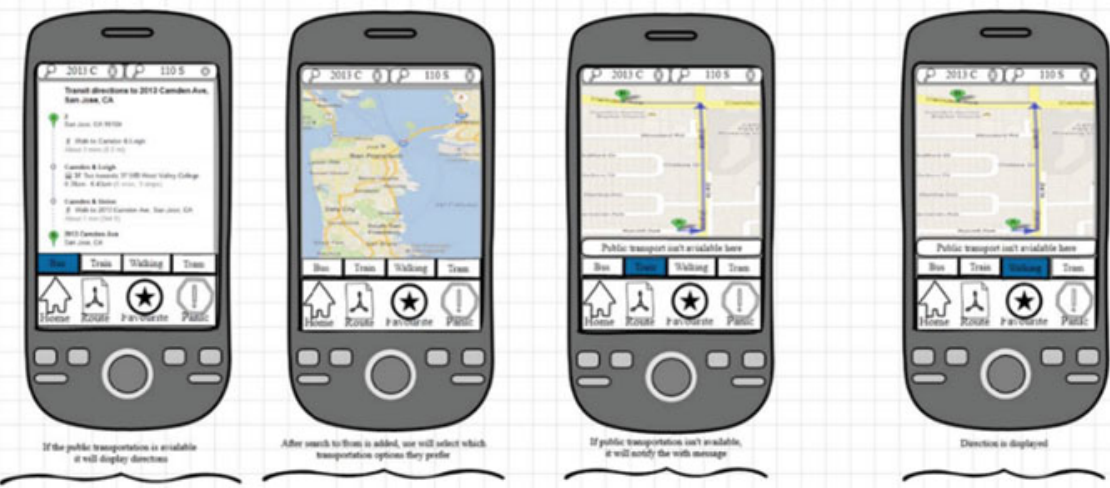

Fig. 5.4 (a) Wireframe (Web). (b) Wireframe (Mobile)

(2011) and gathered usability requirements. After the refinement of the initial mock- 331 up based on the new usability requirements from the users, the first prototype 332 (see Fig. 5.5a, b) was developed, in which system functionalities such as error 333 trapping and remediation were deployed to accommodate human error as well as 334 the failure of system components using HTML, Android Studio framework, and its 335 libraries. This prototype had the ability to (i) recognize path errors, (ii) use the user 336 model to do remediation in case of error, and (iii) capture and do something about 337 the battery running out of power or the loss of the network.

The first prototype of the Assistant smartphone application contained two differ- 339 ent views: Navigation and Settings (see Fig. 5.5a, b). For increasing the effectiveness 340 of real-time information delivery systems and acceptability to intervention content 341 without deterring engagement [39], navigation views allowed the user to receive 342 the push notification about "personalized route" generated by either primary or 343 secondary registered user on the web client. 


\section{Author's Proof}

Once the route is accepted, it is shown as a list (see Fig. 5.5b). Further, the 345 application has the ability to identify the users' location, context (i.e., whether 346 the user is walking, user is waiting for a vehicle, or user is on a vehicle), and 347 real-time information from public transportation. For example, if the user misses 348 the destination stop or waypoint or major delays, the application informs the user 349 through a pop-up showing, "You have missed your bus stop, please get off at the 350 next stop." Once the user gets off from the bus stop, the Assistant application 351 automatically recalculates the user's current location and destination and shows the 352 alternative faster route.

The Settings view allows users to change settings for screen brightness, Blue- 354 tooth (on/off), volume (increase/decrease), and route planner (on/off). In case of 355 emergency, users were able to call their near ones with call buttons.

After the implementation of the first prototype, we conducted new evaluation 357 studies using three usability methods to gather the usability needs of the primary 358 users. During the evaluation process, a user performed various tasks assigned by 359 the evaluators. As an example, some of the findings from this prototype evaluation 360 concerned the web and mobile user interfaces for the route planning section, such 361 as:

- Textual information which should be put together

- The color of the button background (i.e., red)

- Unnecessary buttons

- A detailed report on how to get from place A to B

- Wanted the system to decide the best routing for them

a
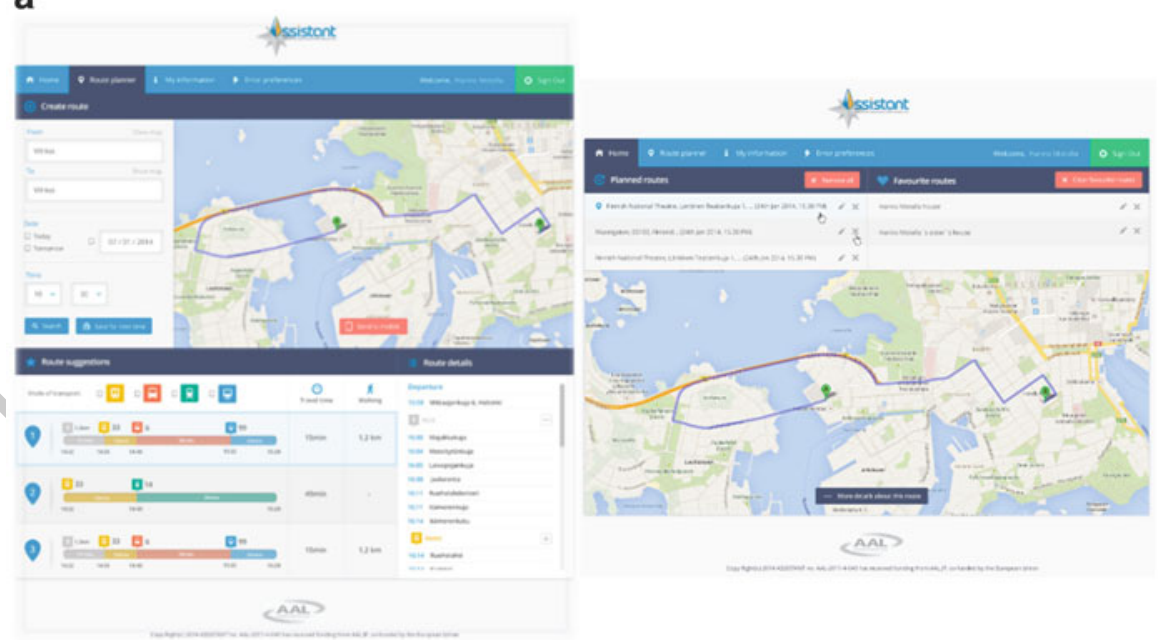

\section{Route planner}

Fig. 5.5 (a) First prototype (Web). (b) First prototype (Mobile). (c) Prototype phase (Mobile) 


\section{Author's Proof}

5 Human-Centered Design Components in Spiral Model to Improve Mobility. . .

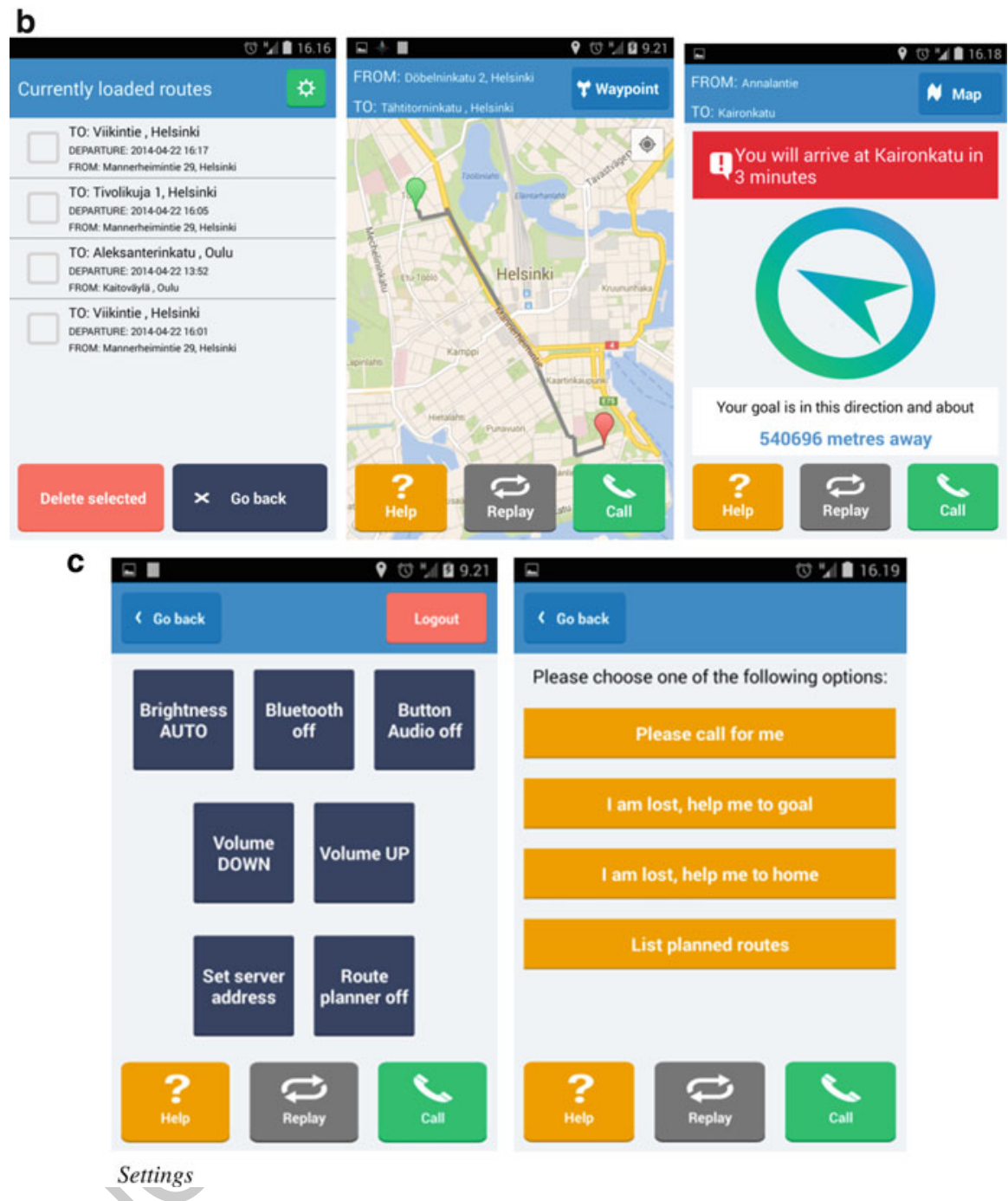

Fig. 5.5 (continued)

To make the Assistant system consistent, user friendly, and usable, we 368 refined the system based on the users' feedback from the evaluation. In the 369 final prototype (see Fig. 5.6a, b), route planner was simplified and removed 370 unnecessary buttons and functionalities of the Assistant system. The application 371 was tested with both primary and secondary users to gather their feedback in 372 real-case scenarios. 


\section{Author's Proof}

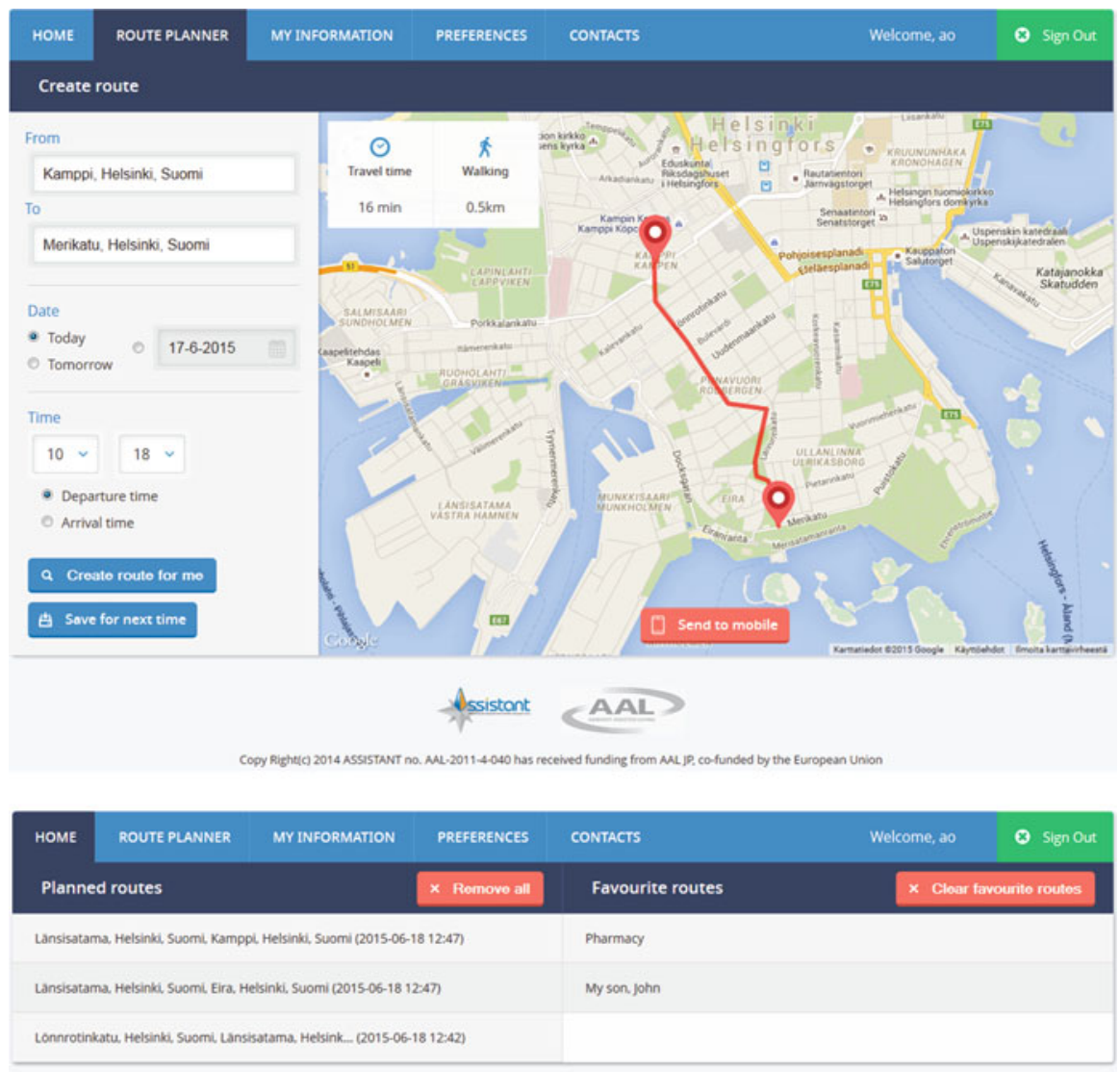

\section{Assistont AAL}

Fig. 5.6 (a) Final prototype (Web). (b) Final prototype (Mobile)

\subsubsection{Usability Evaluation and User Validation}

Usability evaluation methods were employed to get a general overview of the 375 usability issues that were observed during the evaluation process. Usability is 376 measured by the extent to which the intended goals of the use of the overall system 377 are achieved (effectiveness); the resources that have to be expended to achieve the 378 intended goals (efficiency); and the extent to which the user finds the overall system 379 acceptable (satisfaction) [40].

A usability evaluation was performed on the Assistant application to understand 381 how older users experienced each design with their cognitive abilities. To verify 382 the acceptance of the system, older users were recruited to participate in focus 383 


\section{Author's Proof}

5 Human-Centered Design Components in Spiral Model to Improve Mobility...
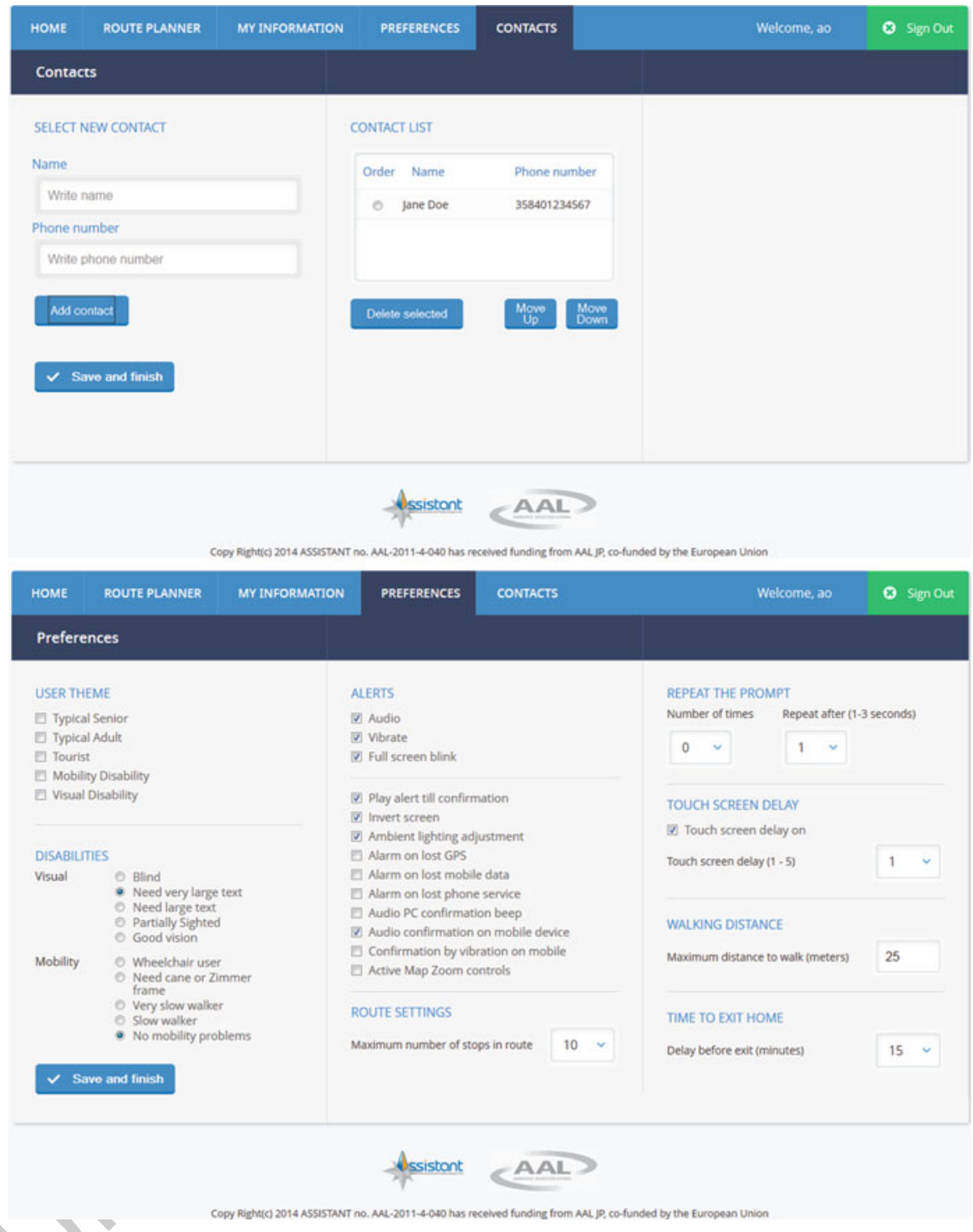

Fig. 5.6 (continued)

groups and evaluations were held in different countries: France, Spain, Austria, 384 and Finland (see Table 5.1). Usability evaluation activities were divided into three 385 phases: concept, prototype, and pilot. Out of three usability method classes proposed 386 by [41], two (i.e., inquiry and inspection) were selected to conduct the usability 387 studies. Various methods were applied because it is difficult to gather usability 388 issues by relying on just one set of data from one specific usability method. The 389 


\section{Author's Proof}

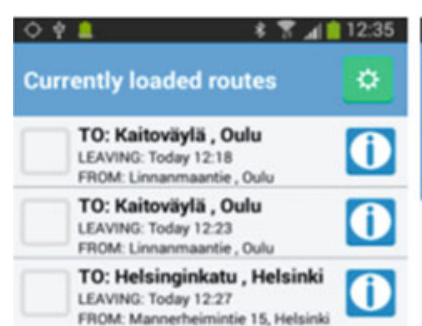

FAOM Mannertheimintie 15, Helsink

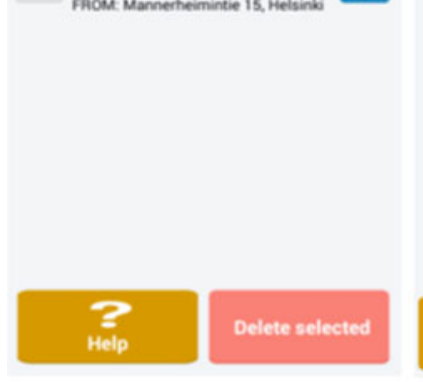

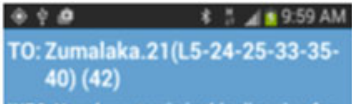

INF O: Your bus stop is in this direction for 2961085 metres.

NEXT LEG: 09:47 bus 33
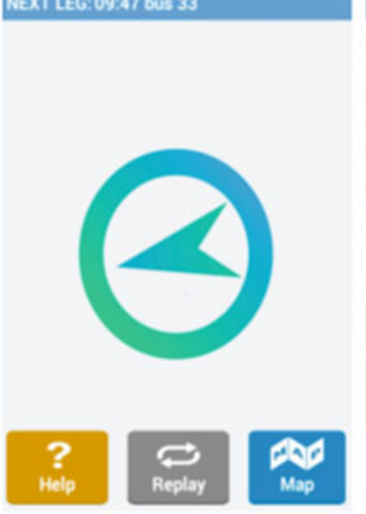

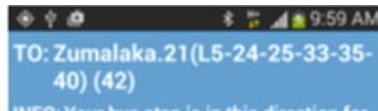

INFO: Your bus stop is in this direction for 2961087 metres.

NEXT LEG: 09:47 bus 33

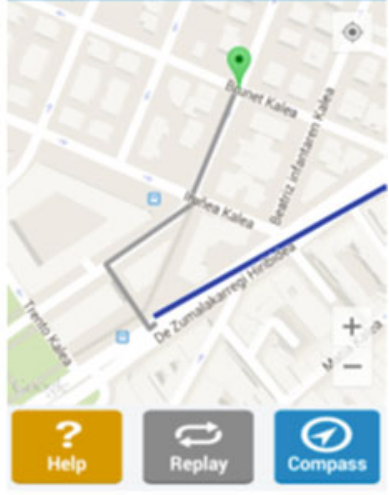

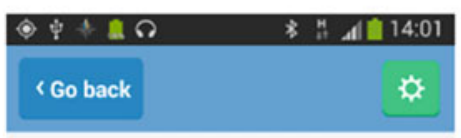

MY CURRENT LOCATION IS:

Linnanmaantie 1290570 Oulu

I think I am lost, please send me a new route
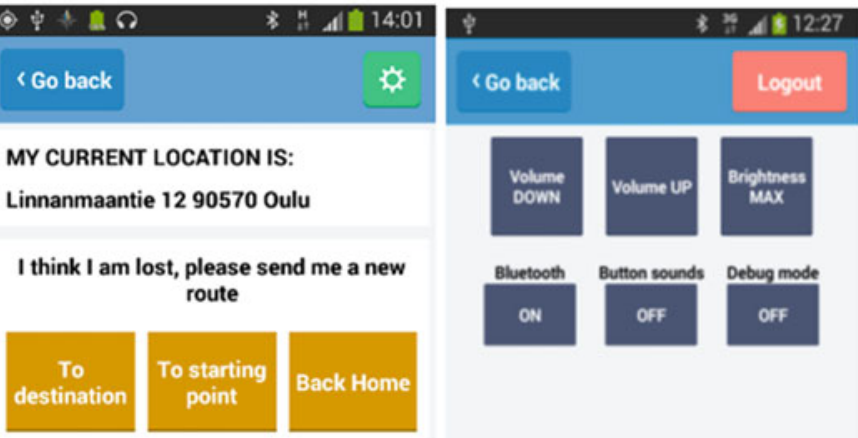

Bluetooth

Button sounds
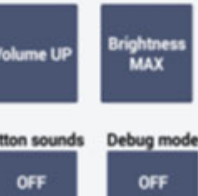

ON

Debug mode

OFF

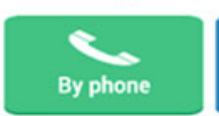

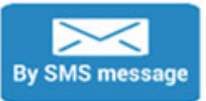

Fig. 5.6 (continued)

method classes included testing, a think-aloud protocol; inspection, a heuristic 390 evaluation; and inquiry, where focus groups, interviews, questionnaires, and diaries 391 were applied. 


\section{Author's Proof}

5 Human-Centered Design Components in Spiral Model to Improve Mobility...

Table 5.1 Summary of the focus groups, evaluation methods, number of participants, and user characteristics from each evaluation phase

\begin{tabular}{|c|c|c|c|c|}
\hline $\begin{array}{l}\text { Evaluation } \\
\text { phase }\end{array}$ & $\begin{array}{l}\text { No. of } \\
\text { participants }\end{array}$ & User characteristics & Country & $\begin{array}{l}\text { Evaluation } \\
\text { method }\end{array}$ \\
\hline $1 \mathrm{st}$ & $\begin{array}{l}4 \\
6 \\
8\end{array}$ & $\begin{array}{l}\text { 1:M, 3:F (65-91/years) } \\
\text { 5:M, 1:F } \\
\text { 4:M, 4:F }\end{array}$ & $\begin{array}{l}\text { France } \\
\text { Spain } \\
\text { Austria }\end{array}$ & $\begin{array}{l}\text { Inquiry } \\
\text { Inspection }\end{array}$ \\
\hline 2nd & $\begin{array}{l}5 \\
5 \\
5\end{array}$ & $\begin{array}{l}\text { 3:M, 1:F, 81,3/years } \\
\text { 3:M, 2:F, 68,4/years } \\
\text { 3:M, 2:F, 68,2/years }\end{array}$ & $\begin{array}{l}\text { Finland } \\
\text { Austria } \\
\text { Spain }\end{array}$ & $\begin{array}{l}\text { Inquiry } \\
\text { Inspection }\end{array}$ \\
\hline $3 \mathrm{rd}$ & $\begin{array}{l}4 \\
5 \\
5\end{array}$ & $\begin{array}{l}\text { 4:M, 0:F, 65-69, 85+ } \\
\text { 1:M, 4:F, 71.4 } \\
\text { 1:M, 4:F, 69,8 }\end{array}$ & $\begin{array}{l}\text { Austria } \\
\text { Spain } \\
\text { Finland }\end{array}$ & $\begin{array}{l}\text { Inquiry } \\
\text { Inspection }\end{array}$ \\
\hline
\end{tabular}

\subsection{Discussion}

Generally, younger groups of users have been given more public and scientific 394 attention than older users when it comes to technology and services [42]. In this 395 research, we conducted a case study where we developed a novel personalized 396 and customized public transportation trip planner for older adults with or without 397 disabilities to show that smartphones can be important tools for them to plan trips 398 and use public transportation more easily. Previous researchers have demonstrated 399 that HCD is a good approach for understanding users and improving the functional 400 and nonfunctional requirements of applications (Zimmermann and Grötzbach, 401 2007) [43]. However, we demonstrated that the HCD design approach is not always 402 applicable when designing an application for a group of older users and requires 403 another framework.

The methodology applied in this study contained four components ((i) analysis, 405 (ii) design, (iii) iterative prototype development and implementation, and (iv) 406 evaluation and user validation) and three phases ((i) concept, (ii) prototype, and (iii) 407 pilot). In the concept phase, initial requirements were gathered from the older users 408 through HCD components. The prototype phase aimed at developing the prototype 409 and improving the design and development based on the feedback from the older 410 users. The pilot phase processed the feedback obtained from the older users during 411 the prototype phase. We explained that interaction is necessary for each phase of the 412 design to enhance usability. Further, the DfF framework approach applied during 413 each iterative cycle of the design and development phase helped the application to 414 detect errors and fix them automatically without any adverse effects on the older 415 users during the user evaluation phase. For example, (i) the system application 416 automatically displays the newer timetable from the public transit system in the 417 case of a missed connection based on the user's location and timetable; (ii) when 418 network coverage is detected as either low or no coverage, the user's caregivers or 419 relatives are notified automatically via the system; and (iii) when the system detects 420 that an older user is walking away from a bus stop, a notification is provided to 421 
the user that he or she is going the wrong way and the correct direction is shown. 422 Having components of HCD, a spiral model, and DfF can provide a tangible design 423 within a short period based on older users' characteristics and desires, which can 424 be constantly evaluated during all phases to receive feedback and transform failure 425 caused by either the user or the system into a success.

As an outcome of the analysis, we discovered that despite having older adults' ${ }_{427}$ involvement throughout the design process with a short iterative cycle, the user 428 could still encounter usability issues and can have an impact in terms of acceptance. 429 For example, similar multiple usability issues were discovered at the end of the 430 pilot phase in multiple countries with different age groups within that group. Some 431 of these issues were (i) using compass on the Assistant application; (ii) voice 432 commands with unclear pronunciation even though the language was in the native 433 language of the user; (iii) using external devices such as Bluetooth headsets; (iv) 434 inaccurate information about the route; (v) location of the buttons; (vi) calibration 435 of the compass; (vii) size and shape of the device; (viii) battery life; (ix) weak 436 GPS signal; (x) data connectivity; (xi) localization of the text; and (xii) auto- 437 lock/screensaver features on the mobile devices, which stopped the users from 438 interacting with the application during their journey. Further, this study also revealed 439 that intermediate older users with a bit of technological knowledge appeared to have 440 fewer usability issues compared to the novice older users who were new to the 441 smartphone devices, which was inconsistent with the previous study by Arfaa and 442 Wang (2014) [46]. The usability needs varied among the older adults with different 443 user characteristics for design. For example, older adults with vision problems had 444 different needs than those with reduced cognitive abilities. We also found out during 445 the study that an application with too much information increases the cognitive load 446 of older users, lowering user satisfaction.

Figure 5.7 represents the data that were analyzed with regard to the perceived 448 use of the Assistant based on a system usability scale (SUS) during the prototype 449 and pilot phases with the same participants. The results indicate that the usability 450 requirements of the older adults changed over time. Our results are consistent 451 with a previous statement from Zajicek (2001): "Requirements for a particular 452 individual vary from time to time due to tiredness or over use of one of the senses 453 e.g. eye strain, and as we know when people age their abilities tend to decrease 454 over time" (pp. 64). Further, our results also showed that there are differences in 455 SUS scores between the three countries where the evaluation was performed. One 456 reason for this is likely to be because of differences (i) in the cultural backgrounds 457 of the older participants, in particular, the users' perceptions of effectiveness, 458 efficiency, and satisfaction [47]. Vatrapu and Perez-Quinones (2006) [48] also 459 established that culture significantly affects usability results and (ii) accessibility 460 of the public transit, in particular, where the application being used was evaluated. 461 For example, the geographic distribution of public transit access, service frequency, 462 transfer distance, and ticketing systems [49] in all three countries is completely 463 different. 


\section{Author's Proof}

5 Human-Centered Design Components in Spiral Model to Improve Mobility...

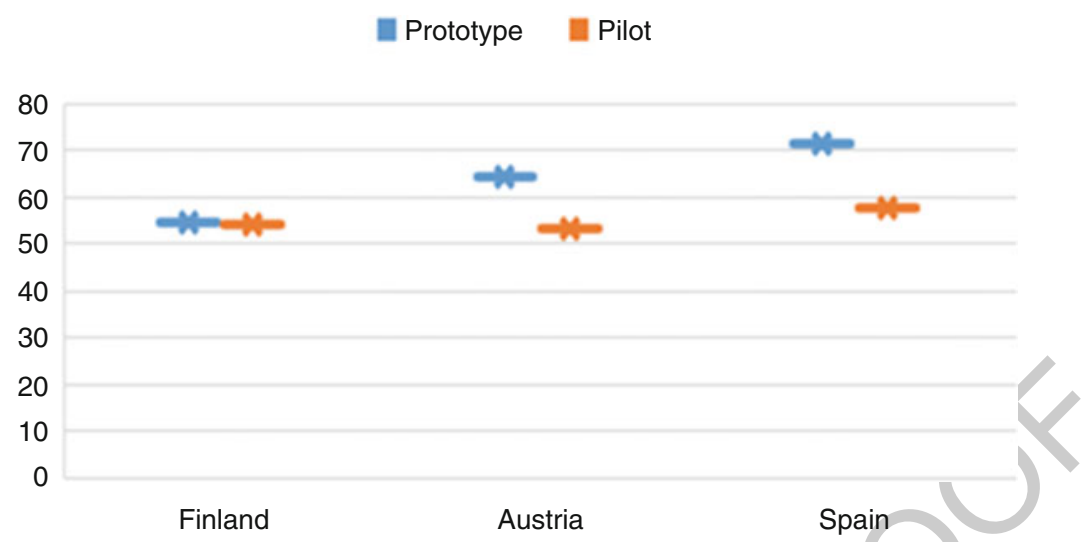

Fig. 5.7 Graphical representation of system usability scale (SUS) between the prototype and pilot phases

Although the SUS scale varied during the prototype and pilot phases, it is 465 noteworthy that the score is still high, which clearly shows that the general 466 perception of the older adults toward the use of the personalized mobile application 467 for public transit was positively high.

First, in the concept phase of the user requirement analysis, the participation of 469 the older adults (65+ years) was quite limited and did not surpass $15 \%$; therefore, 470 potential data bias might exist. However, in the second phase of the project, in both 471 the pilot trials and prototype evaluations, the average age of the older participants 472 was 72.4 years, which could alleviate the prospect of response bias. Second, since 473 all the usability issues collected on the device characteristics (i.e., hardware and 474 software) were based on a single Android-based smartphone device, potential bias 475 might exist regarding the data. Further research with multiple devices is encouraged 476 to gather more usability issues and mitigate the potential data bias. $\quad 477$

We also observed that the satisfaction and usability needs of older users vary with 478 aging; therefore, transit stakeholders (including designers and developers who are 479 developing applications based on open data) must design and develop applications 480 for older adults using the approach of HCD components, a spiral model, and DfF 481 with even shorter iterative cycles to come up with better designs that integrate their 482 needs in a shorter period of time than that we have applied in this study. 483

Certainly, the key findings from this study are beneficial to designers, application 484 developers, researchers, and industries. In fact, the proposed methodology, which 485 we validated with the Assistant platform and older users, can be seen as a method- 486 ology that improves design responses to the usability needs of this heterogeneous 487 group of users. 


\subsection{Conclusion}

In this article, we designed the "Assistant," a web platform and smartphone-based 490 application, for older adult travelers to prepare their personalized journey via public 491 transportation at their convenience and then receive guidance throughout their 492 journey. We presented the framework which consists of HCD with a spiral model 493 and DfF approach during the design process with particular focus on older adults. 494 Additionally, we validated the framework through iterative prototype development 495 and usability testing and final implementation within major European cities in 496 Finland, Austria, and Spain. Our study supports that having the involvement of older 497 adults from the beginning of the design process could initiate a better understanding 498 of their user needs, behavior, and acceptance of transit smartphone applications; 499 these results are in line with the previous study of Hwang and Thorn (1999) [44]. 500 Further, such involvement could also help users feel comfortable and develop a 501 high degree of satisfaction, motivation, and enjoyment regarding these applications' 502 usefulness [45] .

In addition, because our study is related to the number of participants and 504 depends on a specific operating system, the generalizability of the results may 505 be limited, and all stakeholders including both public transportation providers 506 and application developers should take any discussed findings as suggestions for 507 accessibility and usability while designing applications for public transportation 508 rather than as conclusive evidence.

Acknowledgments The results presented in this study are based on "Assistant" project funded by 510 AAL JP, co-funded by the European Union. The authors would like to thank Dr. Stefan Carmien, 511 my colleague in Assistant, for mentoring and for reading and making comments in the earlier 512 versions of this chapter; participating research institutes; funding agencies; and companies from 513 Finland, Spain, Austria, France, and the United Kingdom for their active support throughout the 514 project.

\section{References}

1. Muramatsu N, Akiyama H (2011) Japan: super-aging society preparing for the future. 517 Gerontologist 51(4):425-432 518

2. He W, Goodkind D, Kowal P (2016) An aging world: 2015 international population reports. 519 Aging (Albany, NY) p 165

3. Rosenbloom S, Ståhl A (2003) Automobility among the elderly: the convergence of environ- 521 mental, safety, mobility and community design. Eur J Transp Infrastruct Res 2:197-213 522

4. Sangpikul A (2008) Travel motivations of Japanese senior travellers to Thailand. Int J Tour Res 523 10(1):81-94

5. Jang SC, Wu CME (2006) Seniors' travel motivation and the influential factors: an examination 525 of Taiwanese seniors. Tour Manag 27(2):306-316

6. Patuelli R, Nijkamp P (2016) Travel motivations of seniors. Tour Econ 22(4):847-862 527

7. Carmien S, Dawe M, Fischer G, Gorman A, Kintsch A, Sullivan JF (2005) Socio-technical 528 environments supporting people with cognitive disabilities using public transportation. ACM 529 Trans Comput Interact 12(2):233-262 


\section{Author's Proof}

5 Human-Centered Design Components in Spiral Model to Improve Mobility...

8. Field MJ, Jette AM (2007) The future of disability in America. National Academies Press, 531 Washington, DC

9. Hounsell NB, Shrestha BP, McDonald M, Wong A (2016) Open data and the needs of older 533 people for public transport information. Transp Res Procedia 14(0):4334-4343 534

10. Srichuae S, Nitivattananon V, Perera R (2016) Aging society in Bangkok and the factors 535 affecting mobility of elderly in urban public spaces and transportation facilities. IATSS Res 536 40(1):26-34

11. Marquié J-C, Gabaude C (2010) Aging, transportation and mobility: current issues. Trav Hum 538 73(1):1

12. Barham P, Carmien S, Garzo A (2015) The Assistant Project - Creating a Smartphone App 540 to Assist Older People when Travelling by Public Transport. Proceed 1st Int Conf Inform 541 Commun Technol Ageing Well e-Health 1:253-258

13. Sendra S, Granell E, Lloret J, Rodrigues JJPC (2014) Smart collaborative mobile system for 543 taking care of disabled and elderly People. Mob Networks Appl 19(3):287-302 544

14. Goodman J, Brewster S, Gray P (2004) Connecting elders by facilitating mobility. In: Home 545 Technologies for Connecting Elders, workshop at CHI , 2004, pp 1719-1720 546

15. Plaza I, Martín L, Martin S, Medrano C (2011) Mobile applications in an aging society: status 547 and trends. J Syst Softw 84(11):1977-1988 548

16. Joe J, Demiris G (2013) Older adults and mobile phones for health: a review. J Biomed Inform 549 46(5):947-954

17. Kobayashi M, Hiyama A, Miura T, Asakawa C (2011) Elderly user evaluation of mobile 551 touchscreen interactions much research has been done to evaluate the usability for elderly 552 people of desktop or, Human-Computer Interact - INTERACT 2011 pp. 83-99 553

18. Carmien S (2016) Assistive technology design for intelligence augmentation. Synth Lect Assist 554 Rehabil Heal Technol 5(2):i-171 555

19. Zajicek M (2001) Interface design for older adults. In: Proceedings of the 2001 EC/NSF 556 workshop on universal accessibility of ubiquitous computing: providing for the elderly - 557 WUAUC'01, pp 60-65

20. Zhou X, Zhao S, Chignell M, Ren X (2011) Assessing age-related performance decrements in 559 user interface tasks. In: 2011 IEEE international conference on information and automation, 560 ICIA 2011, pp 817-822 561

21. de Barros AC, Leitão R, Ribeiro J (2014) Design and evaluation of a mobile user interface for 562 older adults: navigation, interaction and visual design recommendations. Procedia Comput Sci 563 27:369-378

22. Rosenbloom S (2009) Meeting transportation needs in an aging-friendly community. Genera- 565 tions 33(2):33-43

23. Wretstrand A, Svensson H, Fristedt S, Falkmer T (2009) Older people and local public transit: 567 mobility effects of accessibility improvements in Sweden. J Transp Land Use 2(2):49-65 568

24. Boehm BW (1988) Spiral model of software development and enhancement. Computer 569 21(5):61-72

25. International Organization for Standardization (1998) ISO 9241-11: ergonomic requirements 571 for office work with visual display terminals (VDTs) - part 11: guidance on usability. Int Organ 572 Stand 2:28 573

26. Kujala S (2003) User involvement: a review of the benefits and challenges. Behav Inf Technol 574 22(1):1-16 575

27. Uzor S, Skelton DA, Baillie L, Skelton DA (2012) Senior designers : empowering seniors to 576 design enjoyable falls rehabilitation tools. In: Proc. SIGCHI Conf. Hum. Factors Comput. Syst， 577 pp 1179-1188 578

28. Ferreira F et al (2013) Elderly centered design for interaction - the case of the S4S medication 579 assistant. Procedia Comp Sci 27:398-408 580

29. Witteman $\mathrm{HO}$ et al (2015) User-centered design and the development of patient decision aids: 581 protocol for a systematic review. Syst Rev 4:11 582

30. Rinkus $S$ et al (2005) Human-centered design of a distributed knowledge management system. 583 J Biomed Inform 38(1):4-17 


\section{Author's Proof}

31. J. Khakurel, J. Porras, and H. Melkas, A systematic literature review on the usability of 585 wearable devices. 2016

32. Lauesen S, Younessi H (1988) Six styles for usability requirements. In: REFSQ, pp 1-12 587

33. Nicolle C, Ross T, Burnett G, Stapleton L (1999) TELSCAN code of good practice and 588 handbook of design guidelines for usability of systems by elderly and disabled drivers and 589 travellers, vol 5

34. Parhi P, Karlson AK, Bederson BB (2006) Target size study for one-handed thumb use on small 591 touchscreen devices. In: Proc. 8th Int. Conf. Human-computer Interact. with Mob. Devices 592 Serv, pp 203-210

35. Creswell JW (2007) Qualitative inquiry \& research design: choosing among five approaches, 594 2nd edn. SAGE Publications

36. ISO/IEC (2001) Guidelines for standards developers to address the needs of older persons and 596 persons with disabilities. In: Iso/Iec 2001, p 36

37. Zhang J (1996) A representational analysis of relational information displays. Int $\mathbf{J}$ Hum 598 Comput Stud 45(1):59-74

38. Hevner AR, March ST, Park J, Ram S (2004) Design science in information systems research. 600 MIS Q 28(1):75-105

39. Morrison LG et al (2017) The effect of timing and frequency of push notifications on usage of 602 a smartphone-based stress management intervention: an exploratory trial. PLoS One 12(1) 603

40. Oppermann R (2002) User-interface design. Handb Inf Technol Educ Train:233-248 604

41. Ivory MY, Hearst MA (2001) The state of the art in automating usability evaluation of user 605 interfaces. ACM Comput Surv 33(4):470-516 606

42. Paul G, Stegbauer C (2005) Is the digital divide between young and elderly people increasing? 607 First Monday 10(10)

43. Zimmermann D, Grötzbach L (2007) Human-computer interaction. Interaction design and 609 usability, vol 4550. Springer/Berlin/Heidelberg, Berlin/Heidelberg

44. Hwang MI, Thorn RG (1999) The effect of user engagement on system success: a meta- 611 analytical integration of research findings. Inf Manag 35(4):229-236 612

45. Khakurel J, Tella S, Penzenstadler B, Melkas H, Porras J (2018) Living with smartwatches 613 and pedometers: the intergenerational gap in internal and external contexts. In: GOODTECHS 614 conference proceedings. P isa, Springer in the Lecture Notes of ICST (LNICST), pp 31-41 615

46. Arfaa J, Wang YK (2014) A usability study on elder adults utilizing social networking sites. In: 616 Lecture notes in computer science (including subseries lecture notes in artificial intelligence 617 and lecture notes in bioinformatics), vol 8518 LNCS, no. PART 2, pp 50-61 618

47. Wallace S, Yu H-C (2009) The effect of culture on usability : comparing the perceptions 619 and performance of Taiwanese and north American MP3 player users. J Usability Stud 4(3): 620 136-146

48. Vatrapu R, Perez-Quinones M (2006) Culture and usability evaluation: the effects of culture in 622 structured interviews. J Usability Stud 1(4):156-170

49. Tyrinopoulos Y, Antoniou C (2008) Public transit user satisfaction: variability and policy 624 implications. Transp Policy 15(4):260-272 


\section{Author's Proof}

\section{AUTHOR QUERIES}

AQ1. Please check usage of "pp." in the text (e.g., "pp. 210"). Should "pp." be changed to "p."?

AQ2. The two paragraphs "In general, the preference ... web platform to a smartphone" have been edited to maintain consistency. Please check and clarify.

AQ3. Please check if the edit to the sentence "Further, application ... transportation" is okay.

AQ4. Please check the use of the phrase "wanted the system... them" for clarity.

AQ5. Please check if the edit to the sentence "therefore, transit stakeholders ... study" is okay.

AQ6. Please provide publisher location for Ref. [35].

AQ7. Please provide page range for Refs. [39, 42]

AQ8. Please provide volume for Ref. [40]. 